\title{
ReporTree: a surveillance-oriented tool to strengthen the linkage between pathogen genetic clusters and epidemiological data
}

\section{Verónica Mixão}

Genomics and Bioinformatics Unit, Department of Infectious Diseases, National Institute of Health Dr. Ricardo Jorge, Av. Padre Cruz, 1600-609 Lisbon, Portugal https://orcid.org/0000-0001-6669-0161

\section{Miguel Pinto}

Genomics and Bioinformatics Unit, Department of Infectious Diseases, National Institute of Health Dr. Ricardo Jorge, Av. Padre Cruz, 1600-609 Lisbon, Portugal https://orcid.org/0000-0001-6370-3051

\section{Daniel Sobral}

Genomics and Bioinformatics Unit, Department of Infectious Diseases, National Institute of Health Dr. Ricardo Jorge, Av. Padre Cruz, 1600-609 Lisbon, Portugal

\section{Adriano Di Pasquale}

National Reference Centre (NRC) for Whole Genome Sequencing of microbial pathogens: data-base and bioinformatics analysis (GENPAT), Istituto Zooprofilattico Sperimentale dell'Abruzzo e del Molise "Giuseppe Caporale" (IZSAM), Teramo, Italy

\section{João Paulo Gomes}

Genomics and Bioinformatics Unit, Department of Infectious Diseases, National Institute of Health Dr. Ricardo Jorge, Av. Padre Cruz, 1600-609 Lisbon, Portugal https://orcid.org/0000-0002-2697-2399

Vitor Borges ( $\square$ vitor.borges@insa.min-saude.pt)

Genomics and Bioinformatics Unit, Department of Infectious Diseases, National Institute of Health Dr. Ricardo Jorge, Av. Padre Cruz, 1600-609 Lisbon, Portugal https://orcid.org/0000-0003-3767-2209

\section{Method Article}

Keywords: ReporTree, genetic clustering, genomic surveillance, public health, automated pipeline

Posted Date: September 30th, 2022

DOl: https://doi.org/10.21203/rs.3.rs-1404655/v2

License: (c) (1) This work is licensed under a Creative Commons Attribution 4.0 International License. Read Full License 


\section{Abstract \\ Background}

Genomics-informed pathogen surveillance strengthens public health decision-making, playing an important role in infectious diseases' prevention and control. A pivotal outcome of genomics surveillance is the identification of pathogen genetic clusters and their characterization in terms of geotemporal spread or linkage to clinical and demographic data. This task often consists of the visual exploration of (large) phylogenetic trees and associated metadata, being time consuming and difficult to reproduce.

\section{Results}

We developed ReporTree, a flexible bioinformatics pipeline that allows diving into the complexity of pathogen diversity to rapidly identify genetic clusters at any (or all) distance thresholds (e.g., high resolution thresholds used for outbreak detection or stable threshold ranges for nomenclature design) and to generate surveillance-oriented reports based on the available metadata, such as timespan, geography or vaccination/clinical status. By handling several input formats (SNP/allele matrices, trees/dendrograms, multiple sequence alignments, VCF files or distance matrices) and clustering methods, ReporTree is applicable to multiple pathogens, thus constituting a flexible resource that can be smoothly deployed in routine surveillance bioinformatics workflows with negligible computational and time costs. This is demonstrated through a benchmarking using core genome- $(\mathrm{cg})$ or whole genome(wg) Multiple Locus Sequence Type (MLST) (cg/wgMLST) datasets of four foodborne bacterial pathogens (each comprising more than a thousand isolates), in which genetic clusters at possible outbreak level were identified and reported in a matter of seconds. To further validate this tool, we reproduced a previous large-scale study on Neisseria gonorrhoeae, demonstrating how ReporTree is able to rapidly identify the main species genogroups and characterize them with key surveillance metadata (e.g, antibiotic resistance data). By providing examples for SARS-CoV-2 and the foodborne bacterial pathogen Listeria monocytogenes, we show how this tool is currently a useful asset in genomicsinformed routine surveillance and outbreak detection of a wide variety of species.

\section{Conclusions}

In summary, ReporTree is a pan-pathogen tool for automated and reproducible identification and characterization of genetic clusters that contributes to a sustainable and efficient public health genomicsinformed pathogen surveillance. ReporTree is implemented in python 3.8 and is freely available at https://github.com/insapathogenomics/ReporTree or as a Docker image at insapathogenomics/reportree.

\section{Impact Statement}


Huge scientific efforts have been made to deploy and standardize bioinformatics solutions that can be integrated in the daily routine genomics surveillance of public health laboratories. Nevertheless, the detection of genetic clusters with possible public health relevance (e.g., outbreak-related samples) is still a challenging step that mostly relies on non-automated (and difficult to reproduce) approaches, such as the visual exploration of (large) phylogenetic trees. Furthermore, there is a current need for flexible bioinformatics solutions enabling an automated integration of genomics and epidemiological/clinical data, which is the ultimate and pivotal step towards an enhanced evidence-informed public-health decision-making. Trying to contribute to fill these gaps, in this article, we present ReporTree, a flexible bioinformatics pipeline suitable for multiple pathogens that identifies samples' genetic clusters in an automated and reproducible way and further characterizes them based on any (and as many) epidemiological or demographical data available, such as timespan, geography or clinical status.

\section{Background}

Whole-genome sequencing (WGS) is the method with the highest resolution to discriminate and classify microorganisms (either at inter- or intra-species level) based on their genetic relatedness. Therefore, the implementation of genomics-informed surveillance systems able to track the circulation of pathogens and monitor their clinical and epidemiologically relevant features is essential for infectious diseases' prevention and control and for a more informed public health decision-making.

Several bioinformatics solutions for the analysis of WGS data are currently available, with most workflows for genetic clustering determination ending-up in the same key output: a phylogenetic tree or a tree-like representation. This often corresponds to a Minimum Spanning Tree (MST) or a dendrogram reflecting the allele distances that result from a core genome- $(\mathrm{cg})$ or whole genome- $(\mathrm{wg})$ Multiple Locus Sequence Type (MLST) analysis (commonly used approach for bacterial pathogens [1]), or to a rooted tree reflecting the Single Nucleotide Polymorphism (SNP) distances that result from a multiple sequence alignment (e.g., as routinely applied for viruses [2], such as SARS-CoV-2 or Monkeypox). Subsequently, the identification and characterization of epidemiologically/biologically relevant genetic clusters (e.g., clusters of outbreak-related strains) often consists of the visual exploration of these (large) phylogenetic trees and associated metadata, taking advantage of robust visualization tools, such as those provided by PHYLOViZ [3], GrapeTree [4], Nextstrain [5], Microreact [6] or Taxonium [7]. As such, this task can be timeconsuming and difficult to reproduce.

In this context, there is a continuous scientific effort to automate the identification of clusters at specific genetic thresholds [4,8-13] and develop dynamic cluster/lineage nomenclature systems, such as the Pango system for SARS-CoV-2 [14], or the bacteria-oriented "SNP address" and "allele hash" of SnapperDB and chewieSnake workflows [9,10], respectively. Still, the field would benefit from the development of automated and more flexible tools that can be used for a wide variety of species, not only to facilitate the detection of genetic clusters at any (or all) distance thresholds of a tree, but also to automatically characterize them based on the available metadata variables of interest. 
Here, we present ReporTree, an automated surveillance-oriented resource that allows diving into the complexity of pathogen diversity to rapidly identify genetic clusters at any distance thresholds between samples and further characterize them according to any relevant epidemiological indicator in a reproducible manner.

\section{Implementation}

ReporTree is a command-line tool implemented in python 3.8 that represents a flexible solution to obtain clustering information at any sample distance thresholds (partitions) either for species that require a $\mathrm{cg} / \mathrm{wgMLST}$ analysis or for those that rely on SNPs/multiple sequence alignments for tree reconstruction. As shown in Figure 1, ReporTree pipeline can be divided into three major steps:

\section{Input processing}

The methodology used for WGS data analysis varies from species to species. For this reason, ReporTree was carefully designed to accept multiple input formats (Table 1), being suitable for application in a wide variety of pathogens. Besides SNP/allele matrices and trees/dendrograms in newick format, ReporTree accepts other input formats such as multiple sequence alignments, VCF files or distance matrices. For instance, when a multiple sequence alignment is provided, ReporTree runs the module alignment_processing.py (also available in standalone mode) to clean the alignment according to the user's specifications and to convert it into a SNP matrix that will be used in the remaining steps. Moreover, when sample genetic variability is provided in the format of multiple VCF files or a list of mutations (variant sites), ReporTree uses vcf2mst [15] to do this format conversion. Besides the input transformation, ReporTree's input processing step can also involve the filtration of the input files to: i) remove samples with excess of missing data (e.g., samples with less than $95 \%$ of cgMLST loci called); ii) remove informative sites/loci from the SNP/allele matrices (e.g., wgMLST loci called in less than $98 \%$ samples); and iii) analyze a subset of samples fulfilling the metadata parameters specified by the user (e.g., samples from a given ST or year) (Figure 1). This dynamic approach allows maximizing the loci/positions shared by a subset of samples, thus contributing to an increased resolution power and, consequently, a higher confidence in the clustering analysis, aligned with a previously explored rationale [16].

\section{Clustering}

Once the input files have been processed, ReporTree determines genetic clusters at all user-defined partition thresholds. When the input file corresponds to a phylogenetic tree, ReporTree runs the module partitioning_treecluster.py (also available in standalone mode), which takes advantage of TreeCluster [8] to automatically determine the genetic clusters using one or several of the different algorithms provided 
by this tool. For all the other input types (except for the distance matrix which can only be used with a hierarchical clustering $[\mathrm{HC}]$ approach), the user can choose between genetic clustering using a GrapeTree or a $\mathrm{HC}$ algorithm (Figure 1). When a GrapeTree algorithm is requested (MSTreeV2 or goeBURST [4,17]), ReporTree runs the module partitioning_grapetree.py (also available in standalone mode), which uses modified version of this program (available at https://github.com/insapathogenomics/GrapeTree), to obtain the MST and all the genetic clusters. When a $\mathrm{HC}$ algorithm is requested, ReporTree runs the module partitioning_HC.py (also available in standalone mode), which calculates pairwise hamming distances with cgmlst-dists (https://github.com/tseemann/cgmlst-dists) and determines the genetic clusters using one or several of the different algorithms provided by SciPy [18]. The main output of each of these three clustering options is always a so-called "partitions table" with clustering information for each sample at all the distance thresholds and clustering algorithms requested by the user.

As a complement, ReporTree can optionally identify ranges of distance thresholds associated with cluster stability, i.e. subsequent partition thresholds in which clustering composition is similar. This kind of analysis is usually a key step in nomenclature design. If requested by the user, ReporTree determines those "stable" regions by running a modified version of the code of the Comparing Partitions tool (http://www.comparingpartitions.info) [19]. This new version (available at https://github.com/insapathogenomics/ComparingPartitions) takes as input the "partitions table" with clustering information at all possible thresholds, and assesses several metrics (Simpson's Index of Diversity, Adjusted Rand and Adjusted Wallace coefficient) to compare the clustering information at consecutive partitions (from “ $n+1$ " to " $n$ "). Based on a previously described approach $[19,20]$, ReporTree then uses the neighborhood Adjusted Wallace coefficient (nAWC) to ultimately determine regions of cluster stability $[16,21]$.

\section{Summary report}

The final step of ReporTree is the generation of summary reports with the metadata_report.py module (also available in standalone mode). Following the user's specifications, this script can perform cluster characterization according to any relevant epidemiological indicator present in the metadata (e.g., source, vaccination status, antibiotic resistance phenotype, etc.). Similar summary reports can be generated to assess the distribution of any (and as many) user-specified variables of interest (e.g., ST distribution by year). When the time variable "date" is provided in the metadata, ReporTree automatically infers other time units (ISO week and ISO year) and metrics (e.g., cluster timespan) relevant for surveillance purposes. Moreover, ReporTree can provide count/relative frequency reports for any grouping variable, such as the relative frequency of the different (sub-)lineages/clusters circulating in the country over time. Noteworthy, ReporTree allows requesting specific reports for sample(s) of interest, as well as the application of filters in the metadata table to select subsets of samples that will be included in the analysis/report (without the need of generating a new subset metadata table). 
Besides these main reports (Figure 1), ReporTree generates multiple parallel outputs that enable a fine exploration of intermediate data (e.g., pairwise distance matrices, filtered alignments, trees/dendrograms), while rendering standardized formats that can be easily explored through multiple compatible visualization tools. For instance, users can interactively visualize and explore the ReporTree derived clusters by uploading an updated metadata table (with cluster information) together with the original/derived newick MST/dendrogram to interactive tools, such as auspice.us [5], Microreact [6] or GrapeTree [4]. In particular, ReporTree outputs can also be uploaded to grapetree-gis (https://github.com/genpat-it/grapetree-gis) to get an interactive visualization of a MST together with temporal and geographical data.

ReporTree is available as a github repository at https://github.com/insapathogenomics/ReporTree or as a docker image at https://hub.docker.com/r/insapathogenomics/reportree.

\section{Results And Discussion}

\section{Benchmarking}

ReporTree benchmarking was performed in a laptop [Intel Core i5(R)] with 16 GB of RAM using four different datasets of distinct foodborne bacterial pathogens: Listeria monocytogenes (1,874 isolates [22]), Salmonella enterica (1,434 isolates [23]), Escherichia coli (1,999 isolate [24]) and Campylobacter jejuni (3,076 isolates [25]). Each of these datasets consists of a collection of genome assemblies and respective allelic profiles of isolates with public sequencing data (deposited in SRA/ENA) that were carefully selected to cover a wide genetic diversity (assessed in terms of Sequence Type [ST] or serotype, depending on the species). Details on the methodology used for the isolates' selection and dataset curation can be found in each dataset repository [22-25]. Briefly, the genome assemblies were performed with Aquamis v1.3.9 [26] using default parameters. cg/wgMLST profiles were determined with chewBBACA v2.8.5 [27] using the 1,748-loci Pasteur cgMLST schema for L. monocytogenes [28], the 8,558-loci INNUENDO wgMLST schema for $S$. enterica, the 7,601-loci INNUENDO wgMLST schema for $E$. coli and the 2,794-loci INNUENDO wgMLST schema for $C$. jejuni [16]. All these schemas were retrieved from chewie-NS (https://chewbbaca.online/stats [29]) in May/June of 2022. As there was the need to determine a set of core loci for $S$. enterica, E. coli and $C$. jejuni datasets, three sets of core loci were obtained for each species with ReporTree by setting distinct "-site-inclusion" thresholds: 0.95, 0.98 and 1.0 (i.e., only keep loci called in at least $95 \%, 98 \%$ and $100 \%$ of the dataset samples). This resulted into cgMLST schemas with 3,261, 3,179 and 874 loci for S. enterica, 2,826, 2,704 and 465 loci for E. coli, and 1,012, 987 and 29 loci for $C$. jejuni, at 0.95, 0.98 and 1.0 thresholds, respectively. As for each of these species, the values obtained at 0.95 and 0.98 were relatively similar, and in the range of what was previously determined for other datasets of the same species [16], the benchmarking proceeded with the loci obtained at the 0.98 threshold. 
To assess the time performance of ReporTree with different dataset sizes, for each species, we generated sub-datasets of randomly selected isolates. The size of these sub-datasets varied between 200 and the maximum number of isolates of the respective dataset (in a 200-isolates step), with ten replicates being run per sub-dataset size. ReporTree was run for each replicate setting '--loci-called 0.95' (i.e. including only samples with at least $95 \%$ of loci called) and requesting summary reports at all possible partition levels obtained with: i) GrapeTree analysis (MSTreeV2 algorithm); and ii) HC analysis (singlelinkage algorithm). Additional runs were performed for each dataset using all isolates but requesting summary reports only for thresholds of "stability" regions (using nAWC, as described above) or at potential "outbreak" level (according to previously described cut-offs) [16,30].

As shown in Figure 2A, for each dataset, ReporTree running time increases linearly with the number of samples, with GrapeTree MSTreeV2 algorithm taking slightly more time than HC single-linkage. Moreover, as expected, a higher number of loci also led to an increased running time. An interesting observation regards the comparison of $S$. enterica and $E$. coli results. Indeed, although the $E$. coli analysis involved a lower number of loci and only a slightly higher number of samples, its running times were higher than those observed for $S$. enterica. This is related to a consistent higher number of clusters in $E$. coli than $S$. enterica dataset at the same threshold (Figure 2B), showing that, as expected, the dataset diversity also impacts ReporTree running times. Overall, using the whole dataset, ReporTree identified and characterized clusters determined at all possible distance thresholds with single-linkage and MSTreeV2 in around 5 and $6 \mathrm{~min}$ for L. monocytogenes, 10 and $11 \mathrm{~min}$ for S. enterica, 18 and $20 \mathrm{~min}$ for $E$. coli and 5 and $7 \mathrm{~min}$ for C. jejuni, respectively. Nevertheless, in a routine surveillance scenario in which genetic clusters are obtained at a single or very few number of distance thresholds (e.g., thresholds for potential outbreak detection), ReporTree running times considerably decreased in all the species (also using the whole dataset) to less than $45 \mathrm{sec}$ with the $\mathrm{HC}$ algorithm and less than $1 \mathrm{~min} 30 \mathrm{sec}$ with the GrapeTree algorithm (Figure $2 \mathrm{C}$ ), reinforcing its suitability for implementation in routine surveillance.

\section{Reproducing a large-scale study on genetic clustering and linkage to antibiotic resistance data in Neisseria gonorrhoeae}

Our team has recently performed an extensive genomics analysis of the bacterial pathogen Neisseria gonorrhoeae [31]. In this study, 3,791 N. gonorrhoeae genomes from isolates collected across Europe were analyzed with a cgMLST approach. Genetic clusters were determined with the goeBURST algorithm implemented in PHYLOViZ [3,12,17,32] for all possible allelic distance thresholds (partitions). Cluster concordance between subsequent distance thresholds was assessed with the nAWC in order to determine regions of cluster stability $[16,19-21]$ that were used for nomenclature purposes and identification of genogroups. The association between metadata and genetic clusters was then performed by timeconsuming table handling with a spreadsheet program. This corresponded to a non-automated workflow and, in the particular case of the cluster congruence analysis and the integration of genetic and clinically or epidemiologically relevant data, it represented a highly demanding process difficult to be applied in 
real-time pathogen surveillance. As such, to validate ReporTree and demonstrate how it can enhance bacterial pathogens' surveillance and research, we used the same dataset as in the previous study [31] and attempted to reproduce the main study outputs with this tool. As shown in ReporTree's Wiki (https://github.com/insapathogenomics/ReporTree/wiki/), using as input the allele matrix with 822 loci [33] and the associated metadata (available in Supplementary material 1 of [31]), ReporTree automatically identified the genetic clusters at all possible partition thresholds of the generated MST and identified the same regions of cluster stability as Pinto et al. [31]. Moreover, it provided an updated metadata table with clustering information at the first partition of each stability region, which could be used as input for visualization in GrapeTree [4]. Furthermore, summary reports with statistics/trends associated with each genetic cluster of low and high levels of stability (i.e. 40 allele differences at the lower level and 79 allele differences at the higher level, similarly to what was found by Pinto et al.) were reported. Finally, ReporTree was able to associate and report the distribution of genetic determinants of antimicrobial resistance in $N$. gonorrhoeae for the different genetic clusters. Importantly, this example allowed a clear validation of the tool by rigorously reproducing the data presented, for example, in Figures 1a, $1 \mathrm{~b}$ and 3 and in Tables 1 and 2 of the previous publication [31]. All these outputs (and additional ones) are available for consultation at ReporTree github repository [34]. Noteworthy, this proof of concept was made with a single command line that ran for approximately $1 \mathrm{~min} 39 \mathrm{sec}$ in a laptop [Intel Core i5(R)] with $16 \mathrm{~GB}$ of RAM.

\section{ReporTree and its application to genomics-informed routine surveillance (e.g., SARS-CoV-2) and outbreak detection (e.g., Listeria monocytogenes)}

Genomics-informed surveillance of SARS-CoV-2 has had an important role in worldwide public health and political decision-making in the last two years. In Portugal, weekly reports of nationwide sequencing surveys are provided to public health authorities and the general public describing important indicators and trends of the evolution and geotemporal spread of the virus [35]. Therefore, after ReporTree validation, we implemented this tool in the routine genomics surveillance of SARS-CoV-2 in the country with the objective of speeding-up the association between genomic and epidemiological data and the generation of the surveillance-oriented reports. For instance, besides its comprehensive usage for monitoring the relative frequency of variants of concern (VOCs) at regional and national levels, ReporTree is applied to identify clusters of high-closely related viruses (e.g., using TreeCluster [8] max-clade or avgclade models at high-resolution levels) that may represent local transmission networks or even superspreading events. An example of ReporTree application in the context of SARS-CoV-2 genomic epidemiology is provided at ReporTree's Wiki [36].

ReporTree can be useful to a broad spectrum of species. One of the most direct and intuitive applications is the analysis of $\mathrm{cg} / \mathrm{wgMLST}$ data for outbreak investigation, namely for foodborne bacterial pathogens (as shown in the Benchmarking section), as this subtyping method delivers sufficiently high resolution and epidemiological concordance [37]. In ReporTree's Wiki [36], it is provided a simple simulated example 
in which, with a single command line, ReporTree builds a MST from cgMLST data and automatically extracts and reports genetic clusters of $L$. monocytogenes at high resolution levels commonly used for outbreak detection ( $\leq 4$ and $\leq 7$ allelic differences, [30]), as routinely performed in Portugal. These two examples show that ReporTree is a useful asset to rapidly generate summary reports with key data (pathogen genetic clusters) and statistics/trends for routine surveillance and outbreak investigation.

\section{Conclusions}

ReporTree represents an automated and flexible pipeline that can be used for a wide variety of species and that facilitates the detection of genetic clusters and their linkage to epidemiological data, in a concept aligned with "One Health" perspectives. Here, we presented the proof of concept of this tool, showing its ability to quickly report a comprehensive WGS-based genogroup assignment for $N$. gonorrhoeae, based on the identification of the discriminatory genetic thresholds reflecting cluster stability, and the rapid correlation of these genogroups (representing main circulating lineages) with any data of interest, such as antimicrobial resistance data. Furthermore, we have shown how its flexibility contributed to speed up SARS-CoV-2 and L. monocytogenes genomics-informed surveillance in Portugal, facilitating and accelerating the production of surveillance-oriented reports. ReporTree benchmarking ultimately demonstrated that this tool can be smoothly implemented in routine surveillance bioinformatics workflows, with negligible computational and time costs. Although ReporTree is currently available as a command line tool, this resource can easily be integrated in start-to-end platforms for genomics/epidemiological analysis (for instance, it will be soon integrated in the COHESIVE Information System (https://cohesive.izs.it) and INSaFLU platform [38]), thus contributing to a sustainable and efficient public health genomics-informed pathogen surveillance.

\section{List Of Abbreviations}

cgMLST - core-genome Multiple Locus Sequence Type

HC - Hierarchical Clustering

MST - Minimum Spanning Tree

SNP - Single Nucleotide Polymorphism

wgMLST - whole-genome Multiple Locus Sequence Type

WGS - Whole-Genome Sequencing

\section{Declarations}

\section{Availability of data and materials}


The datasets generated and/or analyzed during the current study are available in the Zenodo repository (L. monocytogenes: https://zenodo.org/record/7116878; S. enterica: https://zenodo.org/record/7119735; E. coli: https://zenodo.org/record/7120057; C. jejuni: https://zenodo.org/record/7120166; N. gonorrhoeae: https://zenodo.org/record/3946223) or in ReporTree github repository at https://github.com/insapathogenomics/ReporTree/tree/main/examples.

\section{Competing interests}

The authors declare that they have no competing interests

\section{Funding}

This work was supported by funding from the European Union's Horizon 2020 Research and Innovation programme under grant agreement No 773830: One Health European Joint Programme.

\section{Authors' contributions}

VM, MP, JPG and VB idealized and planned the tool. All authors contributed to the design and refinement of the tool. VM performed the programming and benchmarking. VM, MP and VB performed the tool validation. DS performed the dockerization. VM and VB wrote the manuscript. All authors read and approved the final manuscript.

\section{Acknowledgements}

The authors thank Dr. Holger Brendebach, Dr. Carlus Deneke and Dr. Simon Tausch from the German Federal Institute for Risk Assessment for their support during the genome assembly of the samples used in ReporTree benchmarking. We would also like to thank the National Distributed Computing Infrastructure of Portugal (INCD) for providing the necessary resources to run the genome assemblies. INCD was funded by FCT and FEDER under the project 22153-01/SAICT/2016.

\section{References}

1. Jolley KA, Maiden MCJ. Using multilocus sequence typing to study bacterial variation: prospects in the genomic era. Future Microbiol. 2014;9:623-30.

2. Wohl S, Schaffner SF, Sabeti PC. Genomic Analysis of Viral Outbreaks. Annu Rev Virol. 2016;3:173-95. 
3. Ribeiro-Gonçalves B, Francisco AP, Vaz C, Ramirez M, Carriço JA. PHYLOViZ Online: web-based tool for visualization, phylogenetic inference, analysis and sharing of minimum spanning trees. Nucleic Acids Res. 2016;44:W246-51.

4. Zhou Z, Alikhan N-F, Sergeant MJ, Luhmann N, Vaz C, Francisco AP, et al. GrapeTree: visualization of core genomic relationships among 100,000 bacterial pathogens. Genome Res. 2018;28:1395-404.

5. Hadfield J, Megill C, Bell SM, Huddleston J, Potter B, Callender C, et al. Nextstrain: real-time tracking of pathogen evolution. Bioinformatics. 2018;34:4121-3.

6. Argimón S, Abudahab K, Goater RJE, Fedosejev A, Bhai J, Glasner C, et al. Microreact: visualizing and sharing data for genomic epidemiology and phylogeography. Microb Genom. 2016;2:e000093.

7. Sanderson T. Taxonium: a web-based tool for exploring large phylogenetic trees. bioRxiv. 2022. Available from: http://biorxiv.org/lookup/doi/10.1101/2022.06.03.494608

8. Balaban M, Moshiri N, Mai U, Jia X, Mirarab S. TreeCluster: Clustering biological sequences using phylogenetic trees. PLoS One. 2019;14:e0221068.

9. Dallman T, Ashton P, Schafer U, Jironkin A, Painset A, Shaaban S, et al. SnapperDB: a database solution for routine sequencing analysis of bacterial isolates. Bioinformatics. 2018;34:3028-9.

10. Deneke C, Uelze L, Brendebach H, Tausch SH, Malorny B. Decentralized Investigation of Bacterial Outbreaks Based on Hashed cgMLST. Front Microbiol. 2021;12:649517.

11. Ragonnet-Cronin M, Hodcroft E, Hué S, Fearnhill E, Delpech V, Brown AJL, et al. Automated analysis of phylogenetic clusters. BMC Bioinformatics. 2013;14:317.

12. Francisco AP, Vaz C, Monteiro PT, Melo-Cristino J, Ramirez M, Carriço JA. PHYLOViZ: phylogenetic inference and data visualization for sequence based typing methods. BMC Bioinformatics. 2012;13:87.

13. Lees JA, Harris SR, Tonkin-Hill G, Gladstone RA, Lo SW, Weiser JN, et al. Fast and flexible bacterial genomic epidemiology with PopPUNK. Genome Res. 2019;29:304-16.

14. Rambaut A, Holmes EC, O’Toole Á, Hill V, McCrone JT, Ruis C, et al. A dynamic nomenclature proposal for SARS-CoV-2 lineages to assist genomic epidemiology. Nat Microbiol. 2020;5:1403-7.

15. Di Pasquale A, Radomski N, Mangone I, Calistri P, Lorusso A, Cammà C. SARS-CoV-2 surveillance in Italy through phylogenomic inferences based on Hamming distances derived from pan-SNPs, -MNPs and -InDels. BMC Genomics. 2021;22:782.

16. Llarena A-K, Ribeiro-Gonçalves BF, Nuno Silva D, Halkilahti J, Machado MP, Da Silva MS, et al. INNUENDO: A cross-sectoral platform for the integration of genomics in the surveillance of food-borne 
pathogens. EFSA support publ. Wiley; 2018;15. Available

from: http://doi.wiley.com/10.2903/sp.efsa.2018.EN-1498

17. Francisco AP, Bugalho M, Ramirez M, Carriço JA. Global optimal eBURST analysis of multilocus typing data using a graphic matroid approach. BMC Bioinformatics. 2009;10:152.

18. Virtanen P, Gommers R, Oliphant TE, Haberland M, Reddy T, Cournapeau D, et al. SciPy 1.0: fundamental algorithms for scientific computing in Python. Nat Methods. Springer Science and Business Media LLC; 2020;17:261-72.

19. Carriço JA, Silva-Costa C, Melo-Cristino J, Pinto FR, de Lencastre H, Almeida JS, et al. Illustration of a common framework for relating multiple typing methods by application to macrolide-resistant Streptococcus pyogenes. J Clin Microbiol. 2006;44:2524-32.

20. Severiano A, Pinto FR, Ramirez M, Carriço JA. Adjusted Wallace coefficient as a measure of congruence between typing methods. J Clin Microbiol. 2011;49:3997-4000.

21. Barker DOR, Carriço JA, Kruczkiewicz P, Palma F, Rossi M, Taboada EN. Rapid identification of stable clusters in bacterial populations using the adjusted Wallace coefficient. bioRxiv. bioRxiv; 2018. Available from: http://biorxiv.org/lookup/doi/10.1101/299347

22. Mixão V, Brendebach H, Pinto M, Sobral D, Gomes JP, Deneke C, et al. Genome assemblies and respective cgMLST profiles of a diverse dataset comprising 1,874 Listeria monocytogenes isolates. Zenodo; 2022. Available from: https://zenodo.org/record/7116878

23. Mixão V, Brendebach H, Pinto M, Sobral D, Gomes JP, Deneke C, et al. Genome assemblies and respective $\mathrm{wg} / \mathrm{cgMLST}$ profiles of a diverse dataset comprising 1,434 Salmonella enterica isolates. Zenodo; 2022. Available from: https://zenodo.org/record/7119735

24. Mixão V, Brendebach H, Pinto M, Sobral D, Gomes JP, Deneke C, et al. Genome assemblies and respective wg/cgMLST profiles of a diverse dataset comprising 1,999 Escherichia coli isolates. Zenodo; 2022. Available from: https://zenodo.org/record/7120057

25. Mixão V, Brendebach H, Pinto M, Sobral D, Gomes JP, Deneke C, et al. Genome assemblies and respective wg/cgMLST profiles of a diverse dataset comprising 3,076 Campylobacter jejuni isolates. Zenodo; 2022. Available from: https://zenodo.org/record/7120166

26. Deneke C, Brendebach H, Uelze L, Borowiak M, Malorny B, Tausch SH. Species-Specific Quality Control, Assembly and Contamination Detection in Microbial Isolate Sequences with AQUAMIS. Genes. 2021;12. Available from: http://dx.doi.org/10.3390/genes12050644

27. Silva M, Machado MP, Silva DN, Rossi M, Moran-Gilad J, Santos S, et al. chewBBACA: A complete suite for gene-by-gene schema creation and strain identification. Microb Genom. 2018;4. Available from: http://dx.doi.org/10.1099/mgen.0.000166

Page $12 / 17$ 
28. Moura A, Criscuolo A, Pouseele H, Maury MM, Leclercq A, Tarr C, et al. Whole genome-based population biology and epidemiological surveillance of Listeria monocytogenes. Nat Microbiol. 2016;2:16185.

29. Mamede R, Vila-Cerqueira P, Silva M, Carriço JA, Ramirez M. Chewie Nomenclature Server (chewieNS): a deployable nomenclature server for easy sharing of core and whole genome MLST schemas. Nucleic Acids Res. 2021;49:D660-6.

30. Van Walle I, Björkman JT, Cormican M, Dallman T, Mossong J, Moura A, et al. Retrospective validation of whole genome sequencing-enhanced surveillance of listeriosis in Europe, 2010 to 2015. Euro Surveill. 2018;23. Available from: http://dx.doi.org/10.2807/1560-7917.ES.2018.23.33.1700798

31. Pinto M, Borges V, Isidro J, Rodrigues JC, Vieira L, Borrego MJ, et al. clustering to reveal major European whole-genome-sequencing-based genogroups in association with antimicrobial resistance. Microb Genom. 2021;7. Available from: http://dx.doi.org/10.1099/mgen.0.000481

32. Nascimento M, Sousa A, Ramirez M, Francisco AP, Carriço JA, Vaz C. PHYLOViZ 2.0: providing scalable data integration and visualization for multiple phylogenetic inference methods. Bioinformatics. 2017;33:128-9.

33. Pinto M, Borges V, Isidro J, Rodrigues JC, Vieira L, Borrego MJ, et al. Neisseria gonorrhoeae clustering to reveal major European WGS-based genogroups in association with antimicrobial resistance (cgMLST and MScgMLST schemas, allelic profile matrices and GrapeTree input file). Zenodo; 2020. Available from: https://zenodo.org/record/3946223

34. ReporTree. [last accessed 2022 Sep 28]. Available from: https://github.com/insapathogenomics/ReporTree

35. Diversidade genética do novo coronavírus SARS-CoV-2 (COVID-19) em Portugal. [last accessed 2022 Sep 28]. Available from: https://insaflu.insa.pt/covid19/

36. ReporTree Wiki. [last accessed 2022 Sep 28]. Available from: https://github.com/insapathogenomics/ReporTree/wiki

37. Nadon C, Van Walle I, Gerner-Smidt P, Campos J, Chinen I, Concepcion-Acevedo J, et al. PulseNet International: Vision for the implementation of whole genome sequencing (WGS) for global food-borne disease surveillance. Euro Surveill. 2017;22. Available from: http://dx.doi.org/10.2807/15607917.ES.2017.22.23.30544

38. Borges V, Pinheiro M, Pechirra P, Guiomar R, Gomes JP. INSaFLU: an automated open web-based bioinformatics suite "from-reads" for influenza whole-genome-sequencing-based surveillance. Genome Med. 2018;10:46. 


\section{Tables}

Table 1. Summary of ReporTree input types and respective clustering options, with indication of the main outputs provided by this tool.

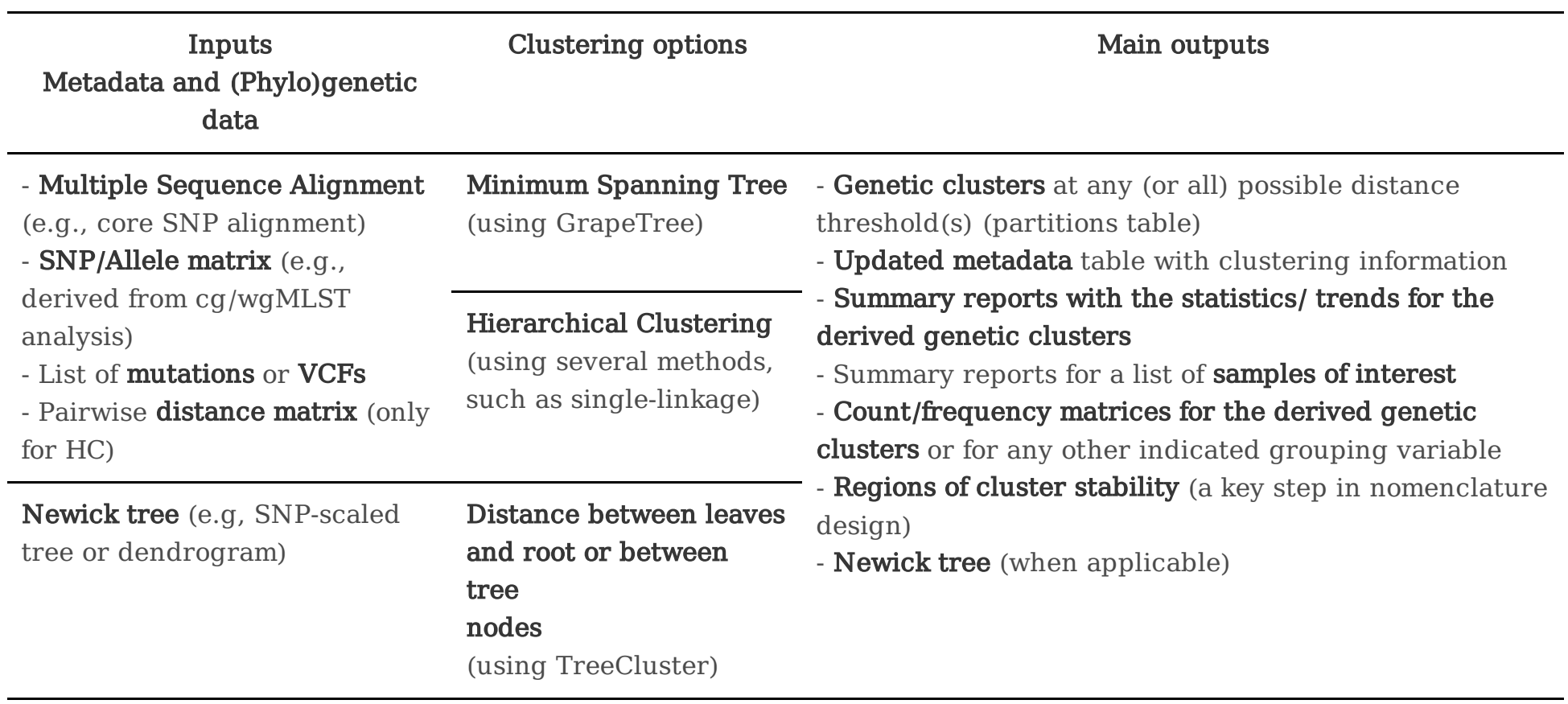

\section{Figures}


METADATA

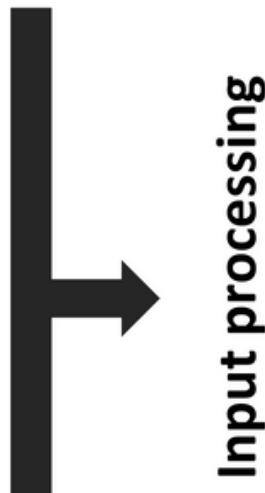

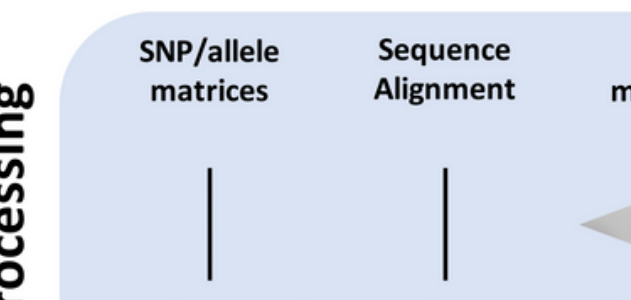

(PHYLO)GENETIC DATA

Filter samples according to metadata (user defined subsets)

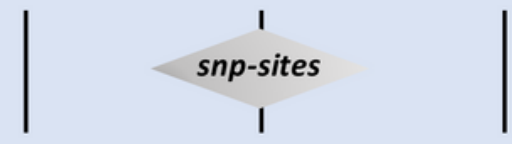

Filter sites/loci and/or samples by missing data

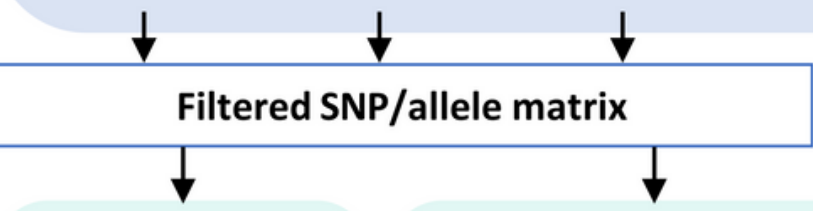

Minimum Spanning Tree

몬

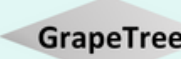

,

MSTreeV2

or

goeBURST

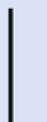

fmst

I

Pairwise Newick

distance tree

matrix
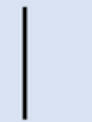

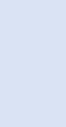

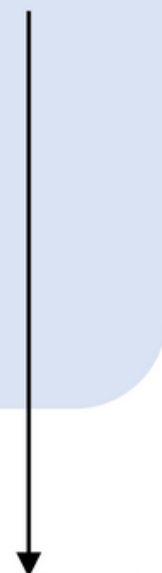

Newick tree

TreeCluster

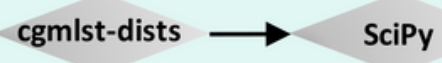

single-linkage

or

other method

avg-clade

or

other method

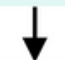

\section{Partitions / Clusters}
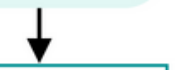
A

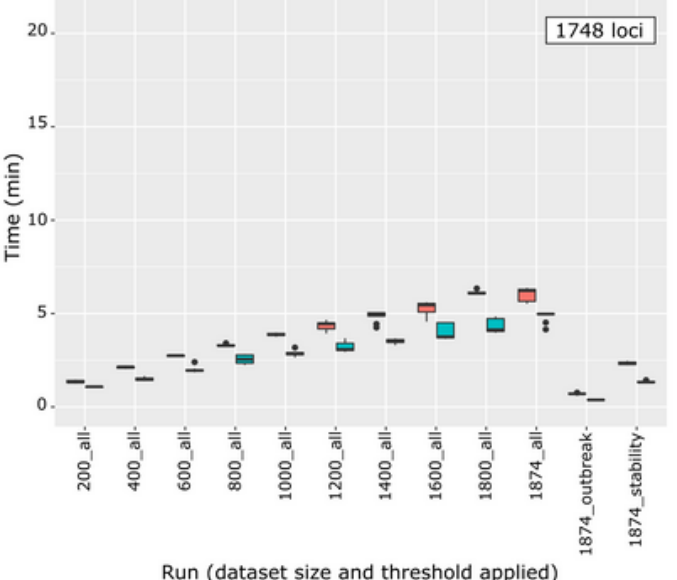

E. coli
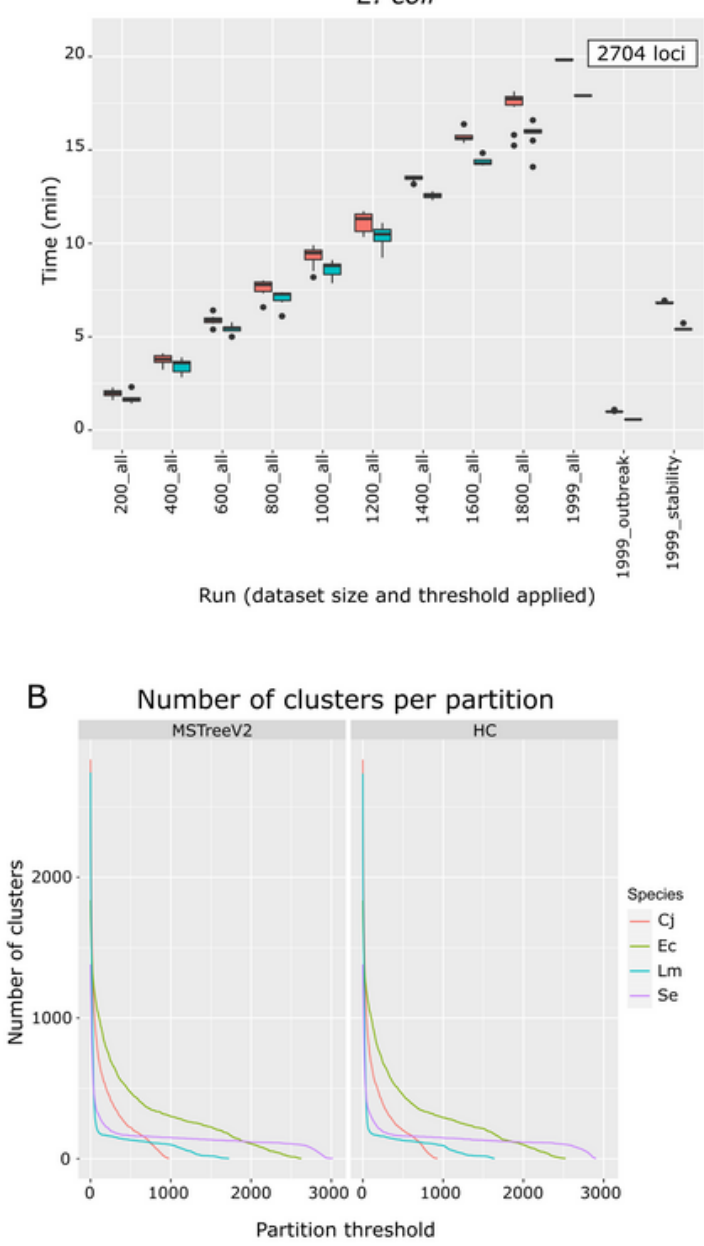

S. enterica

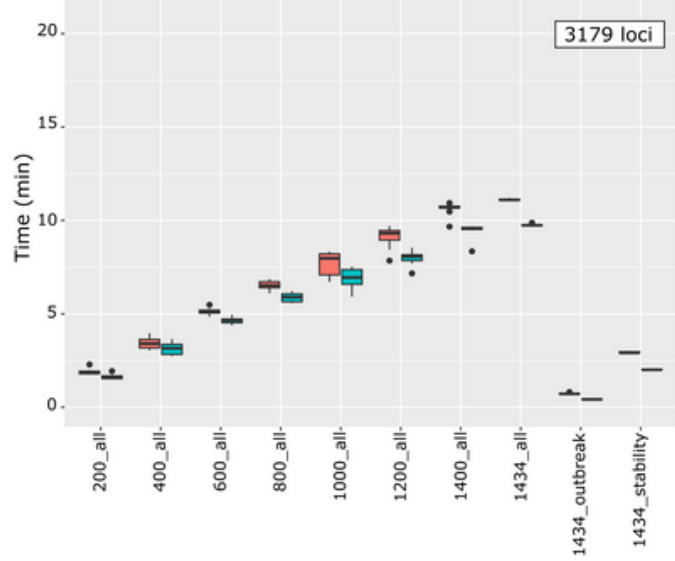

Run (dataset size and threshold applied)

Analysis

白 MSTreev2

它 Single-linkage
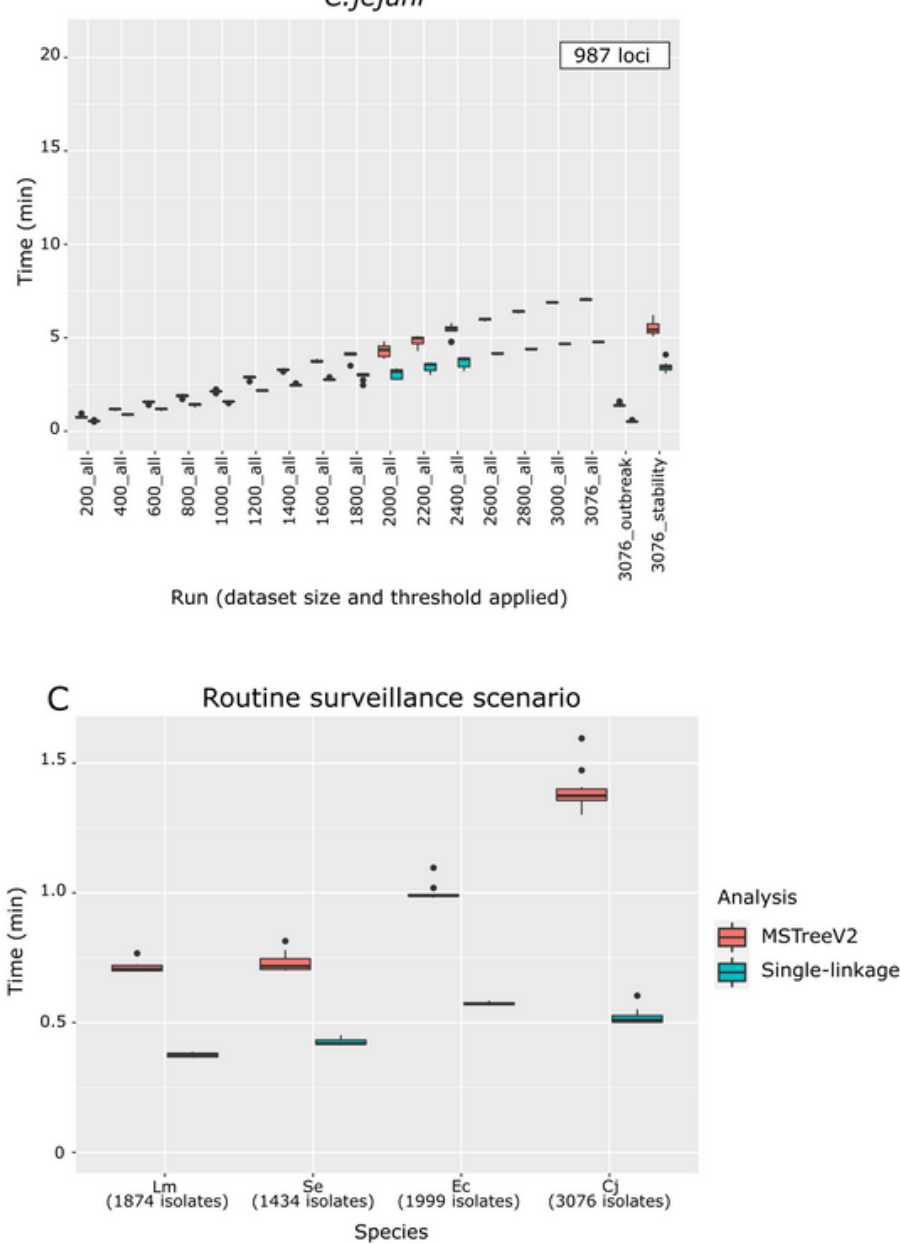

Figure 2

Results of ReporTree benchmarking using datasets for four different species: L. monocytogenes (Lm), S. enterica (Se), Escherichia coli (Ec) and Campylobacter jejuni (Cj). A) ReporTree running times for the 10 replicates of each subset of $L$. monocytogenes (top left), $S$. enterica (top right), $E$. coli (bottom left) and $C$. jejuni (bottom right), where the flag "all" indicates subsets for which ReporTree obtained clusters at all possible thresholds, the flag "outbreak" indicates subsets for which ReporTree obtained clusters at 
potential outbreak level (7 allelic differences for L. monocytogenes, $14(0.43 \%)$ for S. enterica, $9(0.34 \%)$ for $E$. coli and 6 for $(0.59 \%)$ for $C$. jejuni $[16,30]$ ) and the flag "stability" indicates subsets for which ReporTree obtained clusters at all possible thresholds bun only generated reports for those corresponding to stability regions. B) Number of clusters generated at all possible distance thresholds for each dataset. C) Comparison of running times when ReporTree obtained clusters at potential outbreak level. 